\title{
Non-uniformity quantification of temperature and concentration fields by statistical measure and image analysis
}

Dol:

10.1016/j.applthermaleng.2017.06.073

\section{Document Version}

Accepted author manuscript

Link to publication record in Manchester Research Explorer

Citation for published version (APA):

Xiao, Q., Zhai, Y., Lv, Z., Xu, J., Pan, J., \& Wang, H. (2017). Non-uniformity quantification of temperature and concentration fields by statistical measure and image analysis. Applied Thermal Engineering, 124, 1134-1141. https://doi.org/10.1016/j.applthermaleng.2017.06.073

Published in:

Applied Thermal Engineering

\section{Citing this paper}

Please note that where the full-text provided on Manchester Research Explorer is the Author Accepted Manuscript or Proof version this may differ from the final Published version. If citing, it is advised that you check and use the publisher's definitive version.

\section{General rights}

Copyright and moral rights for the publications made accessible in the Research Explorer are retained by the authors and/or other copyright owners and it is a condition of accessing publications that users recognise and abide by the legal requirements associated with these rights.

\section{Takedown policy}

If you believe that this document breaches copyright please refer to the University of Manchester's Takedown Procedures [http://man.ac.uk/04Y6Bo] or contact uml.scholarlycommunications@manchester.ac.uk providing relevant details, so we can investigate your claim.

\section{OPEN ACCESS}




\section{Accepted Manuscript}

Non-uniformity quantification of temperature and concentration fields by statistical measure and image analysis

Qingtai Xiao, Jianxin Pan, Yuling Zhai, Zhihan Lv, Jianxin Xu, Hua Wang

PII:

S1359-4311(17)30960-2

DOI: http://dx.doi.org/10.1016/j.applthermaleng.2017.06.073

Reference: ATE 10592

To appear in:

Applied Thermal Engineering

\section{APPLIED}

THERMAL

ENGINEERING

$\begin{array}{ll}\text { Received Date: } & 15 \text { February } 2017 \\ \text { Revised Date: } & 5 \text { May } 2017\end{array}$

Accepted Date: $\quad 12$ June 2017

Please cite this article as: Q. Xiao, J. Pan, Y. Zhai, Z. Lv, J. Xu, H. Wang, Non-uniformity quantification of temperature and concentration fields by statistical measure and image analysis, Applied Thermal Engineering (2017), doi: http://dx.doi.org/10.1016/j.applthermaleng.2017.06.073

This is a PDF file of an unedited manuscript that has been accepted for publication. As a service to our customers we are providing this early version of the manuscript. The manuscript will undergo copyediting, typesetting, and review of the resulting proof before it is published in its final form. Please note that during the production process errors may be discovered which could affect the content, and all legal disclaimers that apply to the journal pertain. 


\section{Non-uniformity quantification of temperature and concentration fields by statistical measure and image analysis}

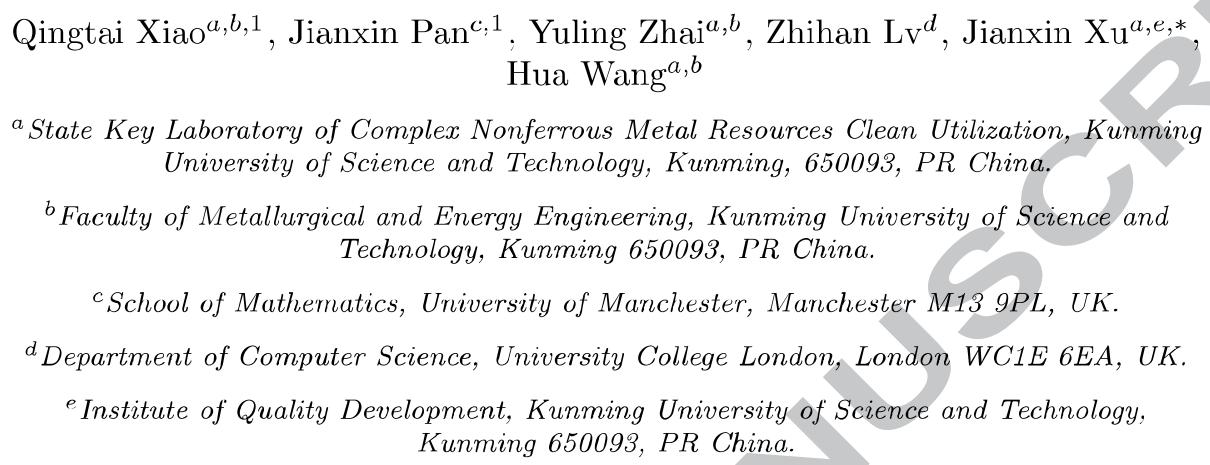
Hua Wang ${ }^{a, b}$

${ }^{a}$ State Key Laboratory of Complex Nonferrous Metal Resources Clean Utilization, Kunming University of Science and Technology, Kunming, 650093, PR China.

${ }^{b}$ Faculty of Metallurgical and Energy Engineering, Kunming University of Science and Technology, Kunming 650093, PR China.

${ }^{c}$ School of Mathematics, University of Manchester, Manchester M13 9PL, UK.

${ }^{d}$ Department of Computer Science, University College London, London WC1E 6EA, UK.

${ }^{e}$ Institute of Quality Development, Kunming University of Science and Technology, Kunming 650093, PR China.

\section{Abstract}

The purpose of this study is to introduce a new technique for quantifying the non-uniformity parameters of temperature and concentration fields. This technique, non-uniformity coefficient (NUC), based on uniform design theory is illustrated by using numerical modeling of microchannel heat sinks with complex structure and computer experiments combined with image analysis. Based on the statistical analogy of star discrepancy, it takes into account the time space and position information (TSPI) of objective. Some of the methods reported in the literature are not found to be able to better characterize and rank very general class of temperature fields correctly. Effectiveness of the new method is

\footnotetext{
${ }^{\mathbf{1}}$ These authors contributed cqually to the paper. *Corresponding author. Tel./Fax.: +86 087165157341.

E-mail addresses: xujianxina@163.com (Jianxin Xu), Jianxin.Pan@manchester.ac.uk (Jianxin Pan) or wanghua65@163.com (Hua Wang).
} 
also demonstrated on the open imaging data of concentration fields. Furthermore, this novel method could be applied to studying a variety of temperature distribution problems in which temperatures are measured with different scales. Keywords: temperature distribution; microchannel heat sinks with complex structure; characterization of field uniformity; star discrepancy; image analysis.

\section{Introduction}

Temperature field is one of the most convenient parameters commonly used to study the flow characteristics in the research of heat transfer enhancement

$4[1,2]$, especially in terms of micro heat sinks with complex structure $[3,4]$.

5 Characterization of temperature ficld uniformity has reccived increasing interest

6 in recent years $[5,6,7]$. However, no single universally and formal quantitative 7 definition of temperature uniformity is hitherto available.

$8 \quad$ One main drawback of classical estimation of temperature field distribution in the most heat transfer investigations is the subjectivity of the measurement interpretation. Indeed, the distribution of temperature field is typically determined with the naked eyes or the researchers' sensory experiences and may yield different results if the same temperature field is in front of different individuals or observed many times by the same operator. It is important to have a quantitative measure of temperature uniformity by computers without hu15 man intervention. Besides, rapidly advancing flow imaging technologies provide 16 powerful tools to help address the above question. Studies in flow visualization, 17 vision measuring and image processing are greatly facilitated by a variety of 18 flow imaging technologies, including electrical resistance tomography [8], posi- 
${ }_{41}^{41}[24]$. The three existing image analysis methods including the standard devia-

tion emission tomography [9], magnetic resonance imaging [10], and electrical capacitance tomography $[11,12,13]$, among other tomographic techniques. During the past many decades, considerable attention has been devoted to apply the technologies to the physical fields [14], such as temperature and concentration ficlds.

A literature survey showed that image analysis has been used to circumvent the subjectivity problem of the concentration field and developed with various degrees of success [15, 16]. For instance, Cabaret et al. (2007) proposed a new technique relying on image analysis to characterize the macro mixing of an impeller in a transparent stirred tank; on the basis of their experimental validations, this method was highly reproducible and robust [17]. Moreover, statistical image analysis is more complicated [18, 19]. For instance, Zhou et al. (2013) developed a general statistical framework for image analysis [20]. They noted an increasing trends and demands of using image processing technology for characterization of subjective human experience [21, 22]. Recently, Fei et al. (2105) and $\mathrm{Xu}$ et al. (2016) presented two straightforward methods (Uniformity Coefficient method and Modified Uniformity Coefficient method based on Uniform Experimental Design) for assessing the bubbles uniformity and mixing quality in a direct-contact heat exchanger. The space-time features of the mixing process have been successfully derived by the former method [23], whereas the later exhibits some advantages such as permutation invariance, rotation invariance (reflection invariance) and the ability to measure projection uniformity 
61 are applied to measure quantitatively for the distribution uniformity of tem${ }_{62}$ perature ficlds. In this work, the main objective is to explore the quantitative

63 characterization of temperature distribution using image analysis.

64

tion method [25], the coefficient of variation method [26] and the image entropy method $[27,28]$ have the inherent advantage of non-intrusive for measuring field uniformity. On the other hand, the approaches remain sensitive to the location of objects in temperature fields. That is to say the time space and position information (TSPI) of objective has not been taken into account in these methods. Hence, further investigations of the engineering characteristics relevant to distribution uniformity of temperature field by means of image analysis are still lacking in a way.

The uniform design proposed by Fang and Wang scatter points uniformly over the experimental domain $[29,30]$. Without loss of generality, suppose there were $s$ factors of interest (dimension of experiment region) over a standard domain $C^{s}=[0,1]$. The key issue was to choose a set of $N$ points $\mathcal{X}=\left\{\boldsymbol{x}_{1}, \cdots, \boldsymbol{x}_{N}\right\}$ such that these points were uniformly scattered on $C^{s}$. Indeed, a measure of uniformity called star discrepancy was adopted, and the goal was to choose $N$ points with the smallest discrepancy $[31,32]$. Hence, the resulting design (uniform design) and has been popularly used during the past more than three decades [33, 34]. Inspired and motivated by Fang et al. (1980, 1995, 2000), Cabaret et al. (2007), Xia et al. (2013), Rodriguez et al. (2014), and Fei et al. (2016), mathematical knowledge and image processing techniques This article is organized as follows. In Section 2, a novel approach of quanti- 
65 fying temperature uniformity is presented; then, temperature data is described

${ }_{66}$ in order to corroborate the validity of the new approach. In Section 3, compar-

67 isons among various uniformity measures for temperature field are performed

${ }_{68}$ using real data and computer experiments. In Section 4, applications to open

69 literature are presented. Finally, summary of findings and concluding remarks 70 are given in Section 5 .

\section{Methodology and temperature dataset}

72 2.1. Proposed method

73 There are a number of existing methods to define the sample points equidistribution, including discrepancy measures, point-to-point measures, and volumetric measures. Results and discussions to be presented in Section 4 will highlight the advantages and deficiency of these existing uniformity measures.

77 This paper proposes a new pixel measure based on the local discrepancy func-

78 tion and image analysis. Mathematically, a digital image is a matrix. A color

79 image can be represented by three gray images. A gray scale image is a matrix

80 with intensity represented as integer values from 0 to 255 . Each matrix element

81 corresponds to a pixel on the image.

Let $F_{u}(\boldsymbol{x})=x_{1} x_{2} \cdots x_{s}$ be the uniform distribution function on $C^{s}=[0,1]^{s}$, where $\boldsymbol{x}=\left(x_{1}, x_{2}, \cdots, x_{s}\right)$. Let $F_{\mathcal{X}}(\boldsymbol{x})$ be the empirical distribution function of $\mathcal{X}=\left\{\boldsymbol{x}_{1}, \boldsymbol{x}_{2}, \cdots, \boldsymbol{x}_{N}\right\}$

$$
F_{\mathcal{X}}(\boldsymbol{x})=\frac{1}{N} \sum_{i=1}^{N} \boldsymbol{1}_{\left[\boldsymbol{x}_{i}, \infty\right]}(\boldsymbol{x})
$$



given by:

$$
D_{p}^{*}(\mathcal{X})=\left[\int_{C^{s}}\left|F_{u}(\boldsymbol{x})-F_{\mathcal{X}}(\boldsymbol{x})\right|^{p} \mathrm{~d} \boldsymbol{x}\right]^{\frac{1}{p}}
$$

For some rectangle region $[0, x]=\left[0, x_{1}\right] \times\left[0, x_{2}\right] \times \cdots \times\left[0, x_{s}\right] \subset C^{s}$, the number of points $N$ encapsulated can be counted. Besides, $\operatorname{card}(\mathcal{X} \cap[\mathbf{0}, \boldsymbol{x}])$ denotes the number of points in the intersection between $\mathcal{X}$ and $[\mathbf{0}, \boldsymbol{x}]$. The local discrepancy function $d_{N}^{*}(\boldsymbol{x})$ at the point $\boldsymbol{x}=\left(x_{1}, x_{2}, \cdots, x_{s}\right)$, therefore, is

$$
\begin{aligned}
d_{N}^{*}(\boldsymbol{x}) & =F_{\mathcal{X}}(\boldsymbol{x})-F_{u}(\boldsymbol{x}) \\
& =\frac{1}{N} \operatorname{card}(\mathcal{X} \bigcap[\mathbf{0}, \boldsymbol{x}])-x_{1} x_{2} \cdots x_{s}
\end{aligned}
$$

90 The following technique is on the basis of image analysis. The color (RGB)

${ }_{91}$ image of temperature field distribution can be converted into a gray-scale image by free ImageJ software. Next, the gray image can be converted into the digital matrix using in-house function of MATLAB software, representing the pixel value of RGB image of mixture.

Definition 1. In this current research, using the mathematical analogy of local discrepancy function, the temperature local discrepancy function (TLDF) of the local rectangular region $\boldsymbol{\theta}=\left[0, \theta_{1}\right] \times\left[0, \theta_{2}\right]$ in a real temperature field is 
given by:

$$
d_{P Q}^{*}(\boldsymbol{\theta})=\frac{\sum_{j=1}^{\theta_{2}} \sum_{i=1}^{\theta_{1}} T_{i, j}}{\sum_{j=1}^{Q} \sum_{i=1}^{P} T_{i, j}}-\frac{\theta_{1} \theta_{2}}{P Q}
$$

${ }_{95}$ where $T_{i j}$ denotes the temperature value at the position $(i, j), \theta_{1} \in\{1, P\}, \theta_{2} \in$ $\{1, Q\}$ and $\frac{\theta_{1}}{\theta_{2}}=\frac{P}{Q}$. Specifically, for the digital temperature image, temperature measurement $T_{i j}$ in the TLDF described in Eq. 4 can be replaced by gray-scale intensity (pixel) value $C_{i j}$.

Here, the TLDF is deemed superior over local discrepancy function due to

the fact that TLDF is adopted to evaluate temperature field uniformity of the whole object surface by infrared thermal imaging yideo camera; where local discrepancy function is point to restricted to a point set, namely temperature measurements at specific point, by thermocouple or pyrometer. This is the main merit of the proposed method. Nevertheless, it is also immediately clear from Eq. 4 that $d_{P Q}^{*}(\boldsymbol{\theta})$ is dependent on the orientation of the first location for calculation. To address this shortcoming, four TLDFs from top-left, bottom-left, bottom-right and top-right orientations, correspond to $q=1,2,3,4$, respectively, are calculated in this work.

Fig. 1 presents a brief illustration of geometric sense of TLDF with above four different start positions. Fig. 1a shows the RGB temperature field and Fig. 1b shows the gray-scale temperature field that both are demonstrated by two digital images with $100 \times 100=10^{4}$ values. Gray-scale images later are converted from the former color file which can consist of real temperature measurements or be RGB temperature image. From Fig. 1c, the two dashed lines (red and pink) represent the local and global rectangle regions, respectively. 
ly smaller. Simply, a smaller $N U C$ denotes uniformly distributed temperature 126 field; while a large $N U C$ indicates poorly distributed temperature field.

The TLDF is obtained easily using the gray intensity level matrix converted from real temperature field.

Definition 2. The single non-uniformity coefficient $\left(N U C_{q}, q=1,2,3,4\right)$ of temperature field distribution is given by:

$$
N U C_{q}=\sup _{\substack{\theta_{1} \in[1, P] \\ \theta_{2} \in[1, Q]}}\left|d_{P Q}^{*}(\boldsymbol{\theta}, q)\right|
$$

where $\left|d_{P Q}^{*}(\boldsymbol{\theta}, q)\right|$ denotes the absolute value of $d_{P Q}^{*}(\boldsymbol{\theta}, q)$. In detail, $N U C_{1}$, $N U C_{2}, N U C_{3}$ and $N U C_{4}$ denote the inhomogeneous degree of temperature distribution in the whole rectangle surface of object, corresponding to top-left, bottom-left, bottom-right and top-right orientations, respectively.

Definition 3. The new index, non-uniformity coefficient (NUC), employed for uniformity cvaluation of temperature field distribution is given by:

$$
N U C=\max \left\{N U C_{1}, N U C_{2}, N U C_{3}, N U C_{4}\right\}
$$

where $\max \{\cdot\}$ denotes the largest value in the set $\{\cdot\}$. In general, if the temperature field is reasonably equidistributed, it is expected that the proportion of the sum of temperature measurements within smaller region is proportionate- 


\subsection{Temperature field description}

Heat transfer enhancement of single phase in micro scale laminar region is one of the most intriguing scientific questions $[3,35]$. Increasing flow area in microchannels is considered to be an effectively method to enhance heat transfer performance, because it is simple, rational and relatively economic [4]. It is generally known that the temperature uniformity of the micro heat sink surface has obvious implications for the lifespan of electronic devices. Special attention also was given to the investigations of flow characteristics and heat transfer [36]. Effectively, besides investigating the liquid flow and heat-transfer characteristics inside the microchannel heat sink, another important thing is to consider the temperature uniformity of the micro heat sink surface. The average fluid temperature and heat transfer coefficient can be used to quantify the fluid flow and temperature distributions [37]. Hence, temperature field is one of the most critical parameters in studies of flow characteristic and heat transfer performance.

On the basis of the Refs. [3], [4], [5] and [6], Fig. 2 presents the geometries of three microchannels: a rectangular micro heat sink, a micro heat sink with triangular reentrant cavities and triangular internal ribs, and a micro heat sink with circular reentrant cavities and circular internal ribs. Moreover, the corresponding conventional rectangular microchannels (smooth micro heat sinks) 47 are considered as reference channels. The geometric characteristics of single microchannel with above three micro heat sinks were described in more detail by Xia et al. and Zhai et al. $[3,4,5,6]$. Temperature fields of fluid of the micro 
heat sinks with different structures were investigated and obtained numerically $[38]$.

It can be clearly seen that the contours of the three kinds of temperature fields are unevenly distributed. They are, moreover, difficult to identify accurately and quantitatively the difference between them. In this current work, non-uniformity coefficient method was proposed to evaluate temperature field distribution uniformity. Ultimately, our aim is to characterize and rank the temperature fields in the whole surface of different microchannels with complex structure.

\section{Results and discussion}

\subsection{Appraisal of different methodologies}

Temperature uniformity measurements are often made visually by the naked eye. This has an inherent degree of subjectivity which inevitably increase the error. Direct imaging analysis routines can remove the subjectivity of the estimation of temperature field uniformity. In this paper, three existing image processing methods, namely, the standard deviation method [25], the coefficient of variation method [26] and the image entropy method [27], are used to verify

the feasibility of our method. In the following discussions, the number of rows is $P=720$ and the number of columns is $Q=1280$ in the recorded images.

In probability theory and statistics, the standard deviation (SD, also represented by the Greek letter sigma $\sigma$ ) is a measure that is used to quantify the amount of variation or dispersion of a set of data values [39]. It is calculated 
as:

$$
S D=\sqrt{\frac{1}{P Q-1} \sum_{k=1}^{P Q}\left(T_{k}-\bar{T}\right)^{2}}
$$

where $T_{k}$ denotes the temperature value in an any-shape region, $\bar{T}=\frac{1}{P Q} \sum_{k=1}^{P Q} T_{k}$ denotes the mean (also called the expected value) of all the temperature measurement. As a result, the smaller the value $S D$ is, the closer the temperature values are to the perfectly equidistribution.

The coefficient of variance $(\mathrm{CoV})$, also known as relative standard deviation (RSD), is a standardized measure of dispersion of probability distribution or frequency distribution. It is often expressed as a percentage, and it is defined as:

$$
C o V=\frac{S D}{\bar{T}}
$$

where $S D$ and $\bar{T}$ are demonstrated by Eq. 7. General speaking, one should use $C o V$ instead of $S D$ for comparison between temperature fields with different scales (e.g., Celsius, Fahrenheit etc.) or widely different means. Nevertheless, when the average temperature $\bar{T}$ is close to 0, the $C o V$ will approach infinity and is therefore sensitive to small changes in the mean $\bar{T}$.

The notion of entropy was used to measure the amount of temperature field information. Assuming that levels of a two-dimensional transient temperature field follow a multinomial distribution, the entropy of the rectangular $P Q$ temperature field is defined as

$$
I E=-P Q \sum_{g=0}^{255} \pi_{g} \log _{2} \pi_{g}
$$


where the theoretical probability $\pi_{g}$ is estimated by the histogram value $h_{g}$. As a result, the valuation criteria of temperature distribution is as follows: if the temperature distribution presents more uniformity, the quantitative value is less. Although $I E$ is available for quantifying the information content of a temperature field, this index cannot be applied to more accurately quantify the field uniformity due to absence of location information of objects.

Some of the notable contributions of this proposed approach (namely, $N U C$ ) over the above existing methods (namely, $S D, C o V$ and $I E$ ) are summarized as follows:

(1) The proposed method, non-uniformity coefficient (NUC) method, generally provides a single number that define the overall quality of a temperature distribution field. A large $N U C$ indicates deviations from uniformity such as local heterogeneity or lopsidedness, while small discrepancy indicates that the temperature is more evenly.

(2) The actual value of the $N U C$ is independent of the unit in which the temperature measurements have been taken, so it is a dimensionless number and can be used for comparison between temperature fields with different scales (e.g., Celsius, Fahrenheit etc.) or widely different means.

(3) The non-uniformity coefficient method takes into account the TSPI of objective. Maybe in some cases, uniformity determined by $S D, C o V$ and $I E$ have a space-time limitation. Particularly, the uniformity with the same $S D$, $C o V$ or $I E$ are very different. 


\subsection{Corroborating the validity of new approach}

As mentioned above, the micro heat sinks with different reentrant cavities and internal ribs have well performance in laminar convective flow and heat transfer. Fig. 3 demonstrates the validity of our method. The image processing illustrated to test the new technique was performed with Matlab using the Image Processing Toolbox (IPT) in this section. Furthermore, the gray-scale images were obtained by transforming the RGB values of each pixel over the whole image to the gray value of it.

As shown in Fig. 3a, it can be clearly scen that the normalized evaluation values (namely, $S D, C o V, I E$ and $N U C$ ) indicate different distributions of fluid temperature in heat sink surface for three microchannels. The smaller of the index, the more uniformity of temperature field in this microchannel heat sink surface. In comparison of three bars of $N U C$, the normalized evaluation value in the microchannels Tri.C-Tri.R and C.C-C.R are smaller than that in the microchannel Rec. (the smooth microchannel without reentrant cavities and internal ribs). So the conclusion can be drawn that the temperature in the main flow zone of the microchannels Tri.C-Tri.R and C.C-C.R tend to be more uniform. To some extent, this is due to the existence of reentrant cavities and internal ribs because they result in a smaller thermal resistance between channel wall and fluid. Furthermore, the microchannel Tri.C-Tri.R has the best heat transfer rate based around one simple fact that small difference in temperature is helpful heat transfer for constant heat flux. Indeed, the present results show some analogies with those obtained by Liu et al. (2012) [40] and 
Zhai et al. (2014) [5]. Closer inspection on other bars in Fig. 3a shows that the proposed index $N U C$ is capable of ranking the temperature pictures in an order that is more agreeable to the mechanism of the heat transfer enhancement in the microchannels; while indexes $S D, C o V$ and $I E$ generate entirely unacceptable ranking orders, respectively.

Fig. 3b shows the distributions of the normalized evaluation values (namely, $S D, C o V, I E$ and $N U C$ ) with velocity for the microchannel heat sink with circular reentrant cavities and circular internal ribs, depending on the range of velocity. Clearly, one can see that $N U C$ are generally reduced as velocity increased in case C.C-C.R. This means that, although the Nusselt number increases with increasing velocity, the distribution uniformity of temperature field becomes better. Compared $S D, C o V$ and $I E$ with $N U C$, it is obviously found that the trend of $S D$ and $C o V$ are similar to that of $N U C$, whereas $I E$ not.

Furthermore, there is more difference in terms of temperature distribution uniformity where high speeds are involved. The results were therefore robust to sensitivity analysis.

Those show that our method has the more accuracy and reliability than the other three existing methodologies. The present approach provides an alternative way to evaluate the distribution uniformity of temperature field.

\subsection{Design for computer experiments}

In above section, we have compared the proposed approach with three existing methods for verifying its feasibility. The comparison was made through temperature distribution analysis of the microchannel data. To provide more 
evidence of the good performance of the proposed method, we carry out intensive computer experiments for accurate estimations of temperature distribution uniformity using MATLAB software. To generate color images for simulating temperature field, the following mathematical formulas are given by:

$$
a+b,-a+b,-a-b, a-b
$$

where $a$ (from 0 to 16 by 0.0125 ) denotes abscissa value while $b$ (from 0 to 9 by 0.0125$)$ denotes ordinate value. Each mathematical formula could generate an RGB image using in-house function pcolor in IPT. Combined these four synthetic RGB images, Fig. 4a and b are respectively employed to investigate uniformity measure of temperature field in two special cases, including locations near the edge and the centre that have the higher temperatures.

Obviously, the simulated temperature fields of Fig. $4 \mathrm{a}$ and $\mathrm{b}$ have the same RGB components, but locations of those pixel points are different. Comparing the variation of resulting values in Table 1 , it is found that there is little difference between the two temperature fields for quantifying their uniformity using the four methods. However, the relative differences 1 (to divide minuend by difference) and 2 (to divide subtrahend by difference) of $N U C$ for these two special cases are both greater, whereas the relative differences of the two uniformity measurements are all close to 0 using other methods, as shown in Table 1. In addition, Fig. 4c depicts the variation with serial-number of local region (or $\boldsymbol{\theta}$ in Eq. 4) of absolute value of TLDF (namely, $\left|d_{P Q}^{*}(\boldsymbol{\theta})\right|$ ) from the 
orientation of top left (or northwest) within the temperature field. It is noticed that the trajectories of $\left|d_{P Q}^{*}(\boldsymbol{\theta})\right|$ present different behavior which is typical for distinguishing different temperature fields uniformity. So based on those results it is concluded that the new method could outperform existing methods and perform more sensitive for practical enginecring application in some sense.

\section{Applications of our method}

\subsection{Open temperature data}

Guo et al. (2011) simulated temperature field under different conditions in the wellhead room of a new auxiliary shaft to find the proper layout and number of radiators that meet wellhead anti-frost requirements, in order to provide guidelines for a practical engineering design [7]. The proposed method offers three corresponding resultant values $N U C=0.0174, N U C=0.1281$, and $N U C=0.0668$ in terms of Fig. 5a (Fig. 4a in Ref. [7]), Fig. 5b (Fig. 6 in Ref. [7]), and Fig. 5c (Fig. 10 in Ref. [7]), respectively. Clearly, the contours of the second one are unevenly distributed while the other two are is uniform, stable and feel comfortable. The $\left|d_{P Q}^{*}(\boldsymbol{\theta})\right|$ of three temperature fields versus $\boldsymbol{\theta}$ from the top-left orientation are illustrated in Fig. 5d. It indicates that $\left|d_{P Q}^{*}(\boldsymbol{\theta})\right|$ frequently fluctuates for small non-uniformity coefficient than great. Those results demonstrate that the proposed $N U C$ is well agreeable to the human perception of temperature uniformity and has a high degree of reliability. Moreover, the mathematical evaluation approach, bringing new insights to quantitative study and compare the temperature field under different conditions, was employed to 
test whether the control temperature meets the control requirement.

\subsection{Other applications of $N U C$}

Accurate determination of the mixing time in reactor is essential for the optimization of mixing processes and minimization of concentration gradients [25].

Fig. 6a clearly shows the 10 volume snapshots of a specific area in the mixing process reported by Rodriguez et al. (2014). They obtained the normalized green index, $G_{i j}^{*}$, and red, $R_{i j}^{*}$, for the point of the reactor at the centre of the specific area. It should be noted that, however, the proposed method was used to obtain a characteristic variation of the pixel intensity in the whole specific area, resulting in a more effective composite index. The variation of the NUC values with horizontal coordinate, $N_{t}$, at the same specific area as in reference [25] was shown in Fig. 6b. The Spearman's rank correlation coefficient $\rho$ between $N U C$ and $R_{i j}^{*}$ is -0.7599 while the $\rho$ between $N U C$ and $G_{i j}^{*}$ is 0.1277 . The former good correlations indicate that $N U C$ represents a meaningful parameter to study the mixing transient as $R_{i j}^{*}$. Furthermore, from a quantifying the mixture distribution point of view, our proposal develops a non-uniformity measure for characterization of subjective human perception but $R_{i j}^{*}$ not and for understanding association between mixed regions and reactor geometry or operating conditions which will be the merit of the proposed approach.

Surface pressure with high spatial resolution is desired in many research scenarios due to that the pressure loads is critical parameters in studies of realworld applications [41]. Fig. 6c shows the pressure field obtained from Ref. [41]. According to Peng and Liu (2016), the jet caused a high pressure region near 
the impingement point and the pressure in the surrounding area was close to ambient pressure. It can be seen that $N U C=0.1081$ and $N U C=0.0966$ were respectively obtained and correspond to above two pressure fields, as shown in Fig. 6c. It indicates that there is little difference of distribution uniformity between them, which is demonstrated that our method was able to extract the quantified information from the experimental data.

\section{Conclusions}

The new conclusions drawn in this current work are summarized as follows:

(1) A novel approach, non-uniformity coefficient method, based on uniform design theory (namely star discrepancy measure) was proposed to measure the uniformity of temperature field. The proposed measure $N U C$ illustrated by image analysis has several notable properties which make it suitable for uniformity characterization in a wide variety of temperature fields with different scales (e.g., Celsius, Fahrenheit etc.) or widely different means. The evaluation value can be used as a benchmark for automatic decision marking.

(2) Effectiveness of the new method is demonstrated on both the presented temperature field data and the real open imaging data. For temperature field, $N U C$ is closely related to Nusselt numbers; For pressure field, $N U C$ distinguishes between relatively more uneven and uniform; For concentration field, $N U C$ is an effective tool to study mixing transient. Considering the location information of objects, it can be seen that the proposed method has the more accurate estimation, with compared with the existing methods previously introduced. 
${ }_{345} a$ Abscissa value

${ }_{346} b \quad$ Ordinate value

\section{Acknowledgements}

\section{Nomenclature}
Abscissa value

(3) It is shown that this technique can be expanded and adapted to analysis measurements gradients in real-world applications, including the temperature field, pressure field, concentration field and velocity field. In general, it is immediately clear that the presented method is only applicable to rectangular region. However, this work presents an alternative route to exploring the field uniformity characterization.

We wish to thank the referees for their numcrous detailed questions and constructive criticism, which greatly improved the presentation. Thanks to Prof. Wuqiang Yang, School of Electrical and Electronic Engineering in The University of Manchester; Prof. Hui Fang, Department of Applied Mathematics in the Huaqiao University; and Prof. Andrea Ducci, Department of Mechanical Engineering in the University College London, for providing valuable discussion and essential help. This work is partially supported by the National Natural Science Foundation of China (Project Nos. 51666006, 51406071 and 51361018), Scientific and Technological Leading Talent Projects in Yunnan Province (Project No. 2015HA019) and Academician Workstation of ZHANG Wenhai in Yunnan Province (Project No. 2015IC005). 
${ }_{347} C$ Standard domain

${ }_{348} \operatorname{card}(A) \quad$ Number of points in area $A$

${ }_{349} C_{i j} \quad$ Gray-scale intensity (pixel)

${ }_{350} \mathrm{CoV}$ Coefficient of variance

${ }_{351} d_{N}^{*}(x) \quad$ Local discrepancy function

${ }_{352} D_{p}^{*}(\mathcal{X}) \quad L_{p}$-discrepancy

${ }_{353} d_{P Q}^{*}(\boldsymbol{\theta}) \quad$ Temperature local discrepancy

${ }_{354} F_{u}(\boldsymbol{x}) \quad$ Uniform distribution function

${ }_{355} F_{\mathcal{X}}(\boldsymbol{x}) \quad$ Empirical distributions function

${ }_{356} g \quad$ Level of pixel

${ }_{357} G_{i j}^{*} \quad$ Normalized green index

${ }_{358} \quad h_{g} \quad$ Histogram value of pixel $g$

${ }_{359} \quad I E \quad$ Entropy of the rectangular ficld

${ }_{360} \quad N \quad$ Number of sample points

${ }_{361} N_{t} \quad$ Horizontal coordinate

$362 N U C$ Non-uniformity coefficient

${ }_{363} p$ Real number

${ }_{364} P$ Number of rows

365 $Q$ Number of columns

366 $q$ Positive integer

${ }_{367} \quad R_{i j}^{*} \quad$ Normalized red index

${ }_{368} s \quad$ Dimension of experiment region

${ }_{369} S D$ Standard deviation 
${ }_{370} \bar{T} \quad$ Mean of all temperature

${ }_{371} T_{i j} \quad$ Temperature value at position $(i, j)$

${ }_{372} T_{k} \quad$ Temperature in an any-shape region

${ }_{373} x_{1} \sim x_{s} \quad$ Coordinates of sample point

${ }_{374} \boldsymbol{x}_{1} \sim \boldsymbol{x}_{s} \quad$ Sample point

${ }_{375} \boldsymbol{\theta} \quad$ Local rectangle region in 2D map

${ }_{376} \theta_{1} \quad$ Positive abscissa value

$377 \quad \theta_{2} \quad$ Positive ordinate value

${ }_{378} \pi_{g} \quad$ Theoretical probability

379 $\rho \quad$ Spearman's rank correlation coefficient

${ }_{380} \mathcal{X} \quad$ Sample points set

\section{References}

[1] W. Cheng, F. Han, Q. Liu, R. Zhao, F. Hanlin, Experimental and theoretical investigation of surface temperature non-uniformity of spray cooling, International Journal of Thermal Sciences 36 (1) (2011) 249-257.

[2] A. A. Hussien, M. Z. Abdullah, M. A. Al-Nimr, Single-phase heat transfer enhancement in micro/minichannels using nanofluids: theory and applications, Applied Energy 164 (2016) 733-755.

[3] G. Xia, L. Chai, M. Zhou, H. Wang, Effects of structural parameters on fluid flow and heat transfer in a microchannel with aligned fan-shaped reentrant cavities, International Journal of Thermal Sciences 50 (3) (2011) $411-419$. 
[4] G. Xia, Y. Zhai, Z. Cui, Numerical investigation of thermal enhancement in a micro heat sink with fan-shaped reentrant cavities and internal ribs, Applied Thermal Engineering 58 (12) (2013) 52-60.

[5] Y. Zhai, G. Xia, X. Liu, Y. Li, Heat transfer in the microchannels with fanshaped reentrant cavities and different ribs based on field synergy principle and entropy generation analysis, International Journal of Heat and Mass Transfer 68 (1) (2014) 224-233.

[6] Y. Zhai, G. Xia, X. Liu, Y. Li, Exergy analysis and performance evaluation of flow and heat transfer in different micro heat sinks with complex structure, International Journal of Heat and Mass Transfer 84 (2015) 293-303.

[7] P. Guo, M. He, Q. Yang, C. Chen, Wellhead anti-frost technology using deep mine geothermal energy, Mining Science and Technology (China) $21(4)(2011) 525-530$.

[8] S. Kim, A. N. Nkaya, T. Dyakowski, Measurement of mixing of two miscible liquids in a stirred vessel with electrical resistance tomography, International Communications in Heat and Mass Transfer 33 (9) (2006) 1088-1095.

[9] S. L. Mckee, R. A. Williams, A. Boxman, Development of solid-liquid mixing models using tomographic techniques, Chemical Engineering Journal and the Biochemical Engineering Journal 56 (3) (1995) 101-107.

[10] Y. Lee, M. J. Mccarthy, K. L. Mccarthy, Extent of mixing in a twocomponent batch system measured using MRI, Journal of Food Engineering 50 (3) (2001) 167-174. 
[11] W. Yang, T. A. York, New ac-based capacitance tomography system, IEE Proceedings - Science Measurement and Technology 146 (1) (1999) 47-53.

[12] W. Yang, L. Peng, Image reconstruction algorithms for electrical capacitance tomography, Measurement Science and Technology 14 (1) (2003) R1-R13(13).

[13] W. Yang, Design of electrical capacitance tomography scnsors, Measurcment Science and Technology 21 (4) (2010) 447-453.

[14] H. Wang, W. Yang, T. Dyakowski, S. Liu, Study of bubbling and slugging fluidized beds by simulation and ect, AIChE Journal 52 (9) (2006) 30783087.

[15] Q. Xiao, S. Wang, Z. Zhang, J. Xu, Analysis of sunspot time series (17492014) by means of 0-1 test for chaos detection, in: International Conference on Computational Intelligence and Security, 2015, pp. 215-218.

[16] Q. Xiao, J. Xu, H. Wang, Quantifying the evolution of flow boiling bubbles by statistical testing and image analysis: toward a general model, Scientific Reports 6 (2016) 31548.

[17] F. Cabaret, S. Bonnot, L. Fradette, , P. A. Tanguy, Mixing time analysis using colorimetric methods and image processing, Industrial and Engineering Chemistry Rescarch 46 (14) (2007) 50325042.

[18] E. Demidenko, Mixed models: theory and applications with R, Wiley, 2013. 
[19] J. Xu, Q. Xiao, Y. Fei, S. Wang, J. Huang, Accurate estimation of mixing time in a direct contact boiling heat transfer process using statistical methods , International Communications in Heat and Mass Transfer 75 (2016) $162-168$.

[20] H. Zhou, L. Li, H. Zhu, Tensor regression with applications in neuroimaging data analysis, Journal of the American Statistical Association 108 (502) (2013) 540-552.

[21] M. A. Lindquist, The statistical analysis of fMRI data, Statistical Science 23 (4) (2009) 439-464.

[22] Q. Xiao, J. Pan, J. Xu, H. Wang, Z. Lv, Hypothesis-testing combined with image analysis to quantify evolution of bubble swarms in a direct-contact boiling heat transfer process, Applied Thermal Engineering 113 (2017) 851 857.

[23] Y. Fei, Q. Xiao, J. Xu, J. Pan, S. Wang, H. Wang, J. Huang, A novel approach for measuring bubbles uniformity and mixing efficiency in a direct contact heat exchanger, Energy 93 (2015) 2313-2320.

[24] J. Xu, Q. Xiao, Y. Chen, Y. Fei, J. Pan, H. Wang, A modified $L_{2}$-star discrepancy method for measuring mixing uniformity in a direct contact heat exchanger, International Journal of Heat and Mass Transfer 97 (2016) 70-76.

[25] G. Rodriguez, T. Anderlei, M. Micheletti, M. Yianneskis, A. Ducci, On the 
measurement and scaling of mixing time in orbitally shaken bioreactors, Biochemical Engineering Journal 82 (3) (2014) 10-21.

[26] A. Amdouni, P. Castagliola, H. Taleb, G. Celano, Onc-sided run rules control charts for monitoring the coefficient of variation in short production runs, European Journal of Industrial Engineering 10 (5) (2016) 639-639.

[27] G. Jumaric, Entropy of markovian processes: application to image entropy in computer vision, Journal of the Franklin Institute 335 (7) (1998) 13271338.

[28] E. Novak, L. M. Paxton, H. J. Tubbs, L. F. Turner, C. W. Keck, J. Yatsu, Assessing phylogenetic relationships of Lycium samples using RAPD and entropy theory, Acta Pharmacologica Sinica 26 (10) (2005) 1217-1224.

[29] K. Fang, The uniform design: application of number-theoretic methods in experimental design, Acta Mathematicae Applicatae Sinica 3 (4) (1980) $363-372$.

[30] Q. Xiao, J. Pan, Z. Lv, J. Xu, H. Wang, Measure of bubble non-uniformity within circular region in a direct-contact heat exchanger, International Journal of Heat and Mass Transfer 110 (2017) 257-261.

[31] K. Fang, W. Shiu, J. Pan, Uniform design based on Latin squares, Statistica Sinica 9 (3) (1995) 905912.

[32] K. Fang, D. K.J. Lin, P. Winker, Y. Zhang, Uniform design: theory and application, Technometrics 42 (3) (2000) 237-248. 
[33] Y. Zhou, K. Fang, An efficient method for constructing uniform designs with large size, Computational Statistics 28 (3) (2013) 1319-1331.

[34] A. M. Elsawah, H. Qin, Asymmetric uniform designs based on mixture discrepancy, Journal of Applied Statistics 43 (12) (2016) 2280-2294.

[35] Y. Zhai, G. Xia, Z. Chen, Z. Li, Micro-PIV study of flow and the formation of vortex in micro heat sinks with cavities and ribs, International Journal of Heat and Mass Transfer 98 (2016) 380-389.

[36] Y. Zhai, G. Xia, X. Liu, J. Wang, Characteristics of entropy generation and heat transfer in double-layered micro heat sinks with complex structure, Energy Conversion and Management 103 (2015) 477-486.

[37] P. Gunnasegaran, H. A. Mohammed, N. H. Shuaib, R. Saidur, The effect of geometrical parameters on heat transfer characteristics of microchannels heat sink with different shapes, International Communications in Heat and Mass Transfer 37 (8) (2010) 1078-1086.

[38] Y. Zhai, Z. Li, H. Wang, J. Xu, Analysis of field synergy principle and the relationship between secondary flow and heat transfer in double-layered microchannels with cavities and ribs, International Journal of Heat and Mass Transfer 101 (2016) 190-197.

[39] J. M. Bland, D. G. Altman, Statistics notes: measurement crror and correlation coefficients, BMJ Clinical Research 313 (7049) (1996) 106-106.

[40] W. Liu, Z. C. Liu, L. Ma, Application of a multi-field synergy principle in 
497

the performance evaluation of convective heat transfer enhancement in a tube, Science Bulletin 57 (13) (2012) 1600-1607.

[41] D. Peng, Y. Liu, A grid-pattern PSP/TSP system for simultaneous pressure and temperature measurements, Sensors and Actuators B Chemical 222 (2015) 141-150. 
Table 1: Performance comparison among the four uniformity measures of temperature distribution in the conducted computer experiments.

\begin{tabular}{ccccc}
\hline Measures & SD & CoV & IE & NUC \\
\hline Special case 1 (Fig. 4a) & 55.4648 & 0.3509 & 7.0781 & 0.0406 \\
Special case 2 (Fig. 4b) & 55.4602 & 0.3509 & 7.0825 & 0.0365 \\
Relative difference 1 (\%) & 0.0083 & -0.0622 & 0 & 10.10 \\
Relative difference 2 (\%) & 0.0083 & -0.0621 & 0 & 11.23 \\
\hline
\end{tabular}




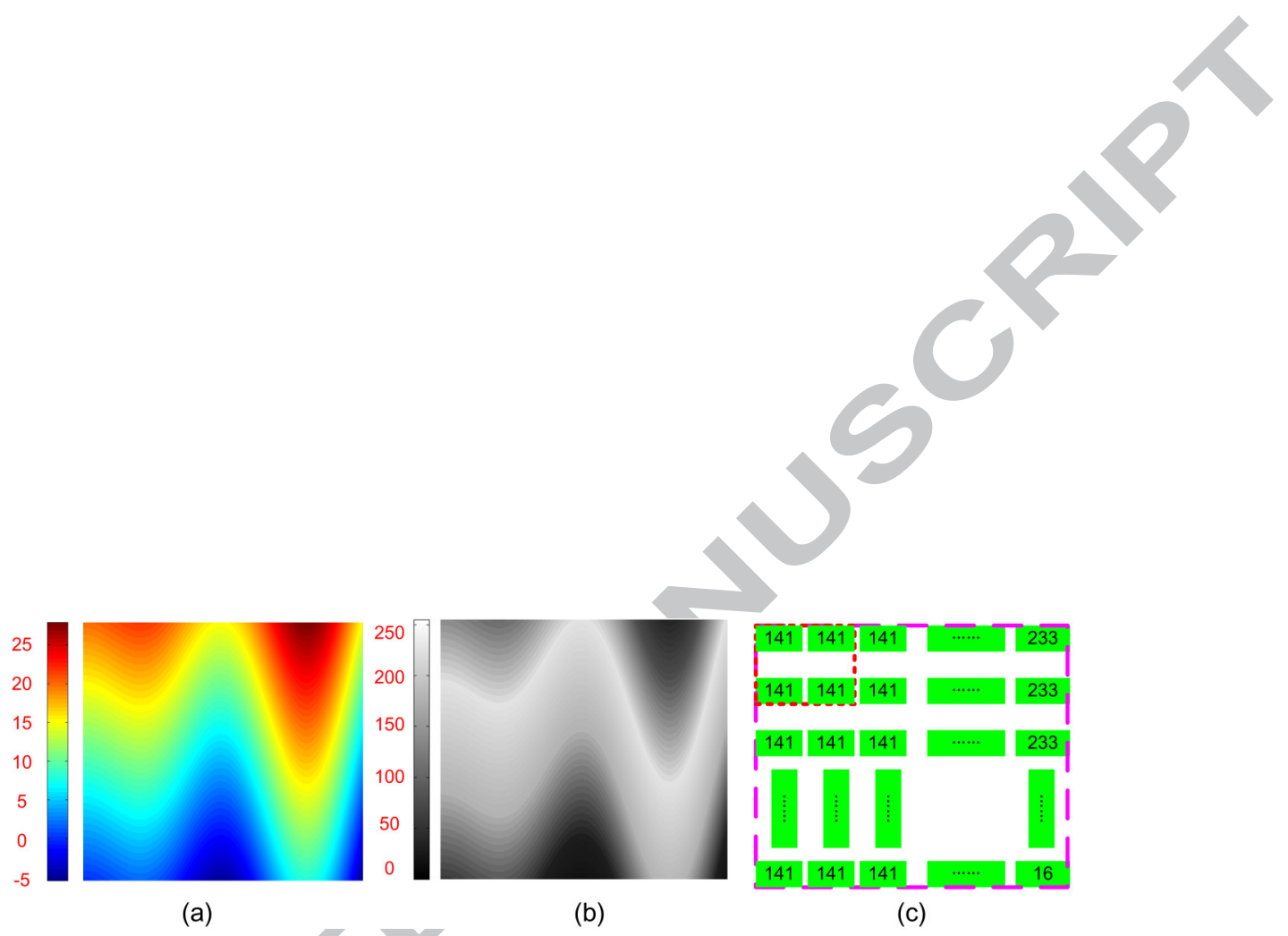

Figure 1: Brief illustration of TLDF with different start position selection: (a) RGB temperature image; (b) gray-scale temperature image; (c) gray intensity level matrix (top-left, bottom-left, bottom-right and top-right orientations correspond to $q=1,2,3,4$, respectively). 


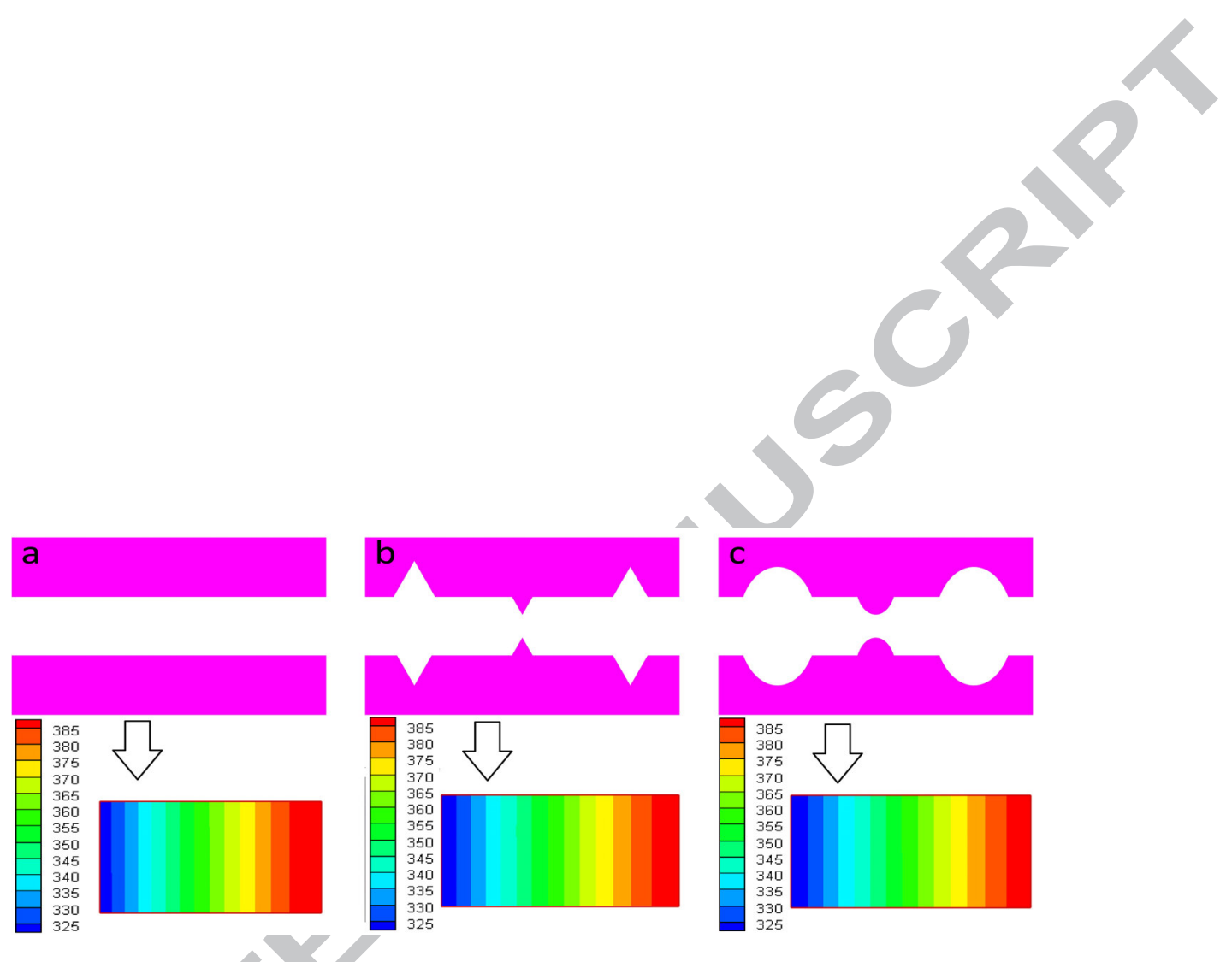

Figure 2: Cross sections of three micro heat sinks (upper) and there corresponding temperature fields in the surface (lower): (a) rectangular microchannel; (b) microchannel with triangular reentrant cavities and triangular internal ribs; (c) microchannel with circular reentrant cavities and circular internal ribs. 

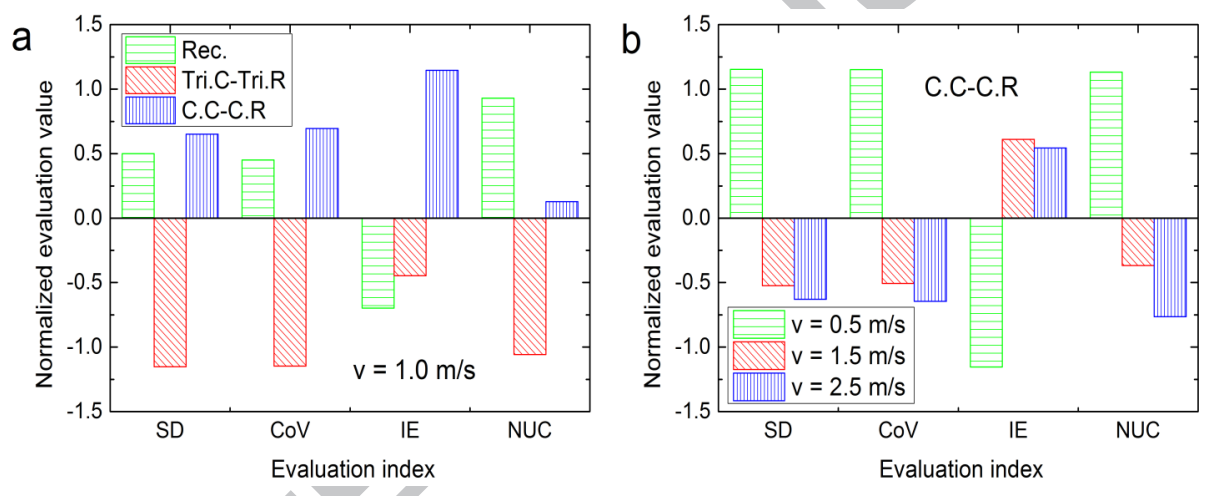

Figure 3: Validation with microchannel structure (a: Rec. denotes the reference microchannel; Tri.C-Tri.R and C.C-C.R denote the two new microchannels) and velocity (b: in terms of a special case C.C-C.R) of different evaluation values of temperature distribution. 

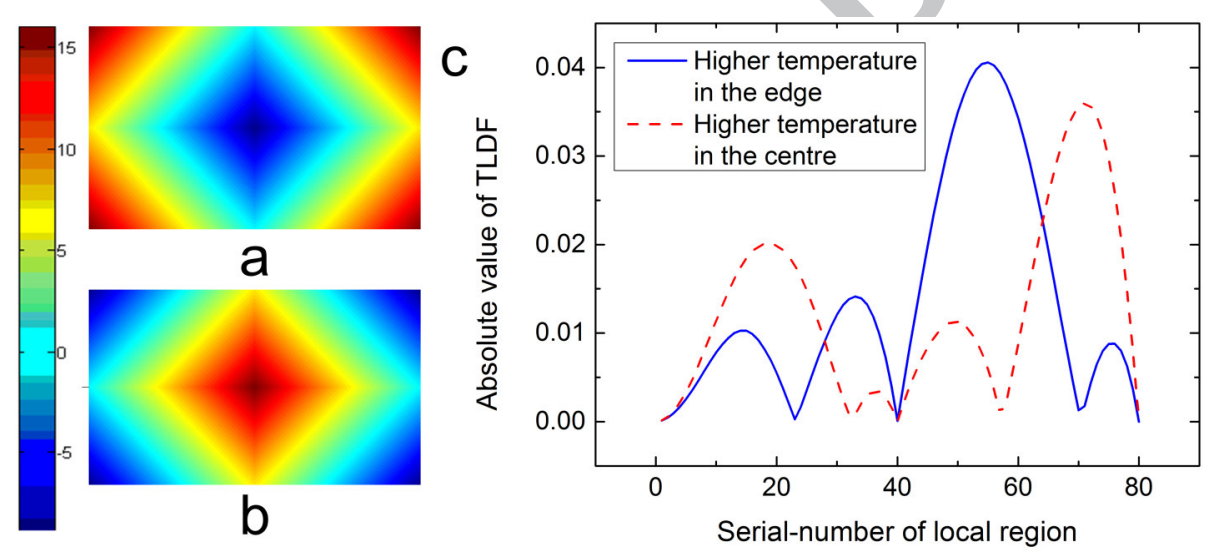

Figure 4: Computer experiments: (a) locations near the edge have the higher temperatures; (b) locations near the centre have the higher temperatures; (c) absolute value of TLDF versus serial-number of local region from the orientation of top left (or northwest). 

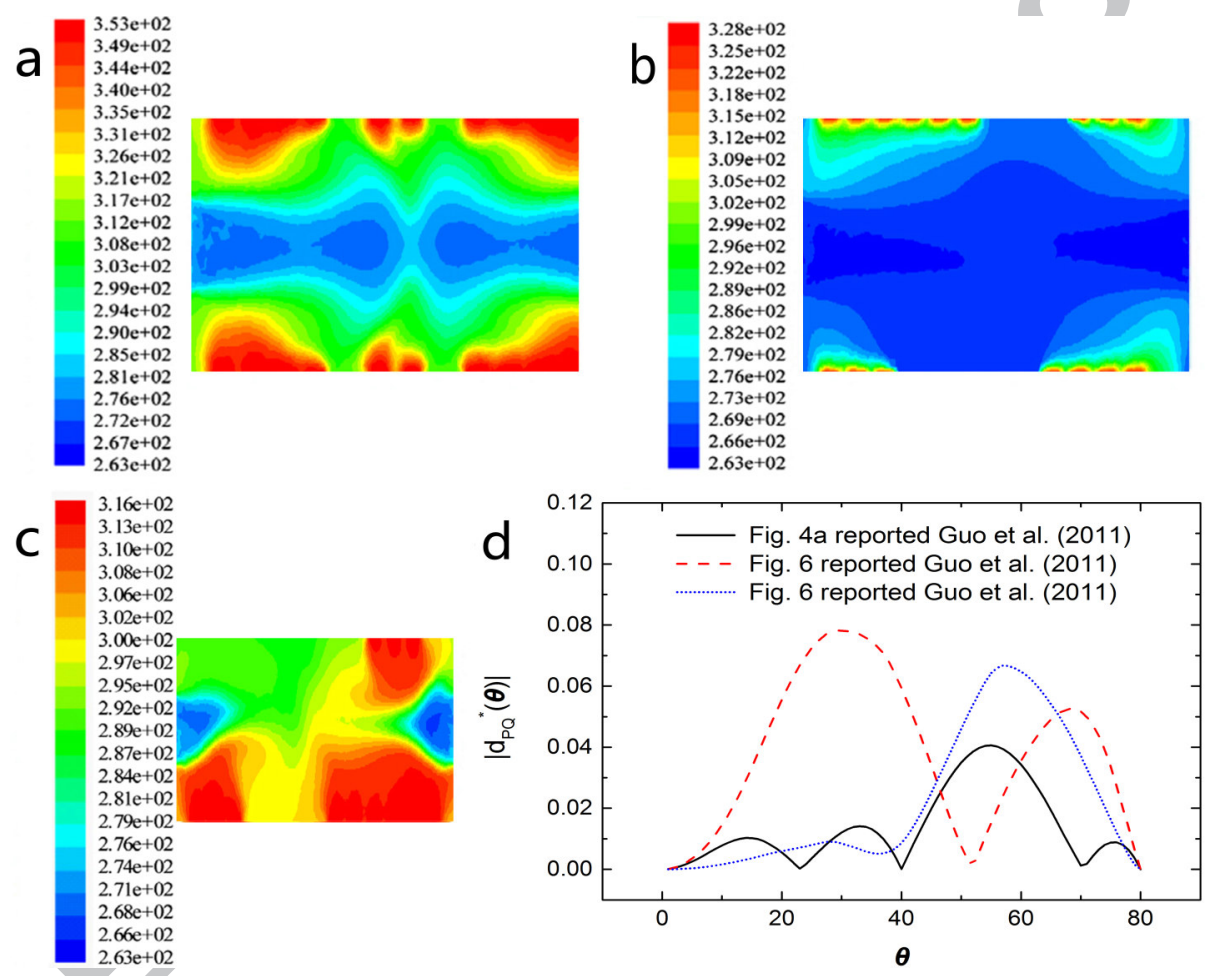

Figure 5: Variation of the $N U C$ values of three reported temperature fields: (a) Fig. 4a in Ref. [7]; (b) Fig. 6 in Ref. [7]; (c) Fig. 10 in Ref. [7] and (d) variation of evaluation values. 

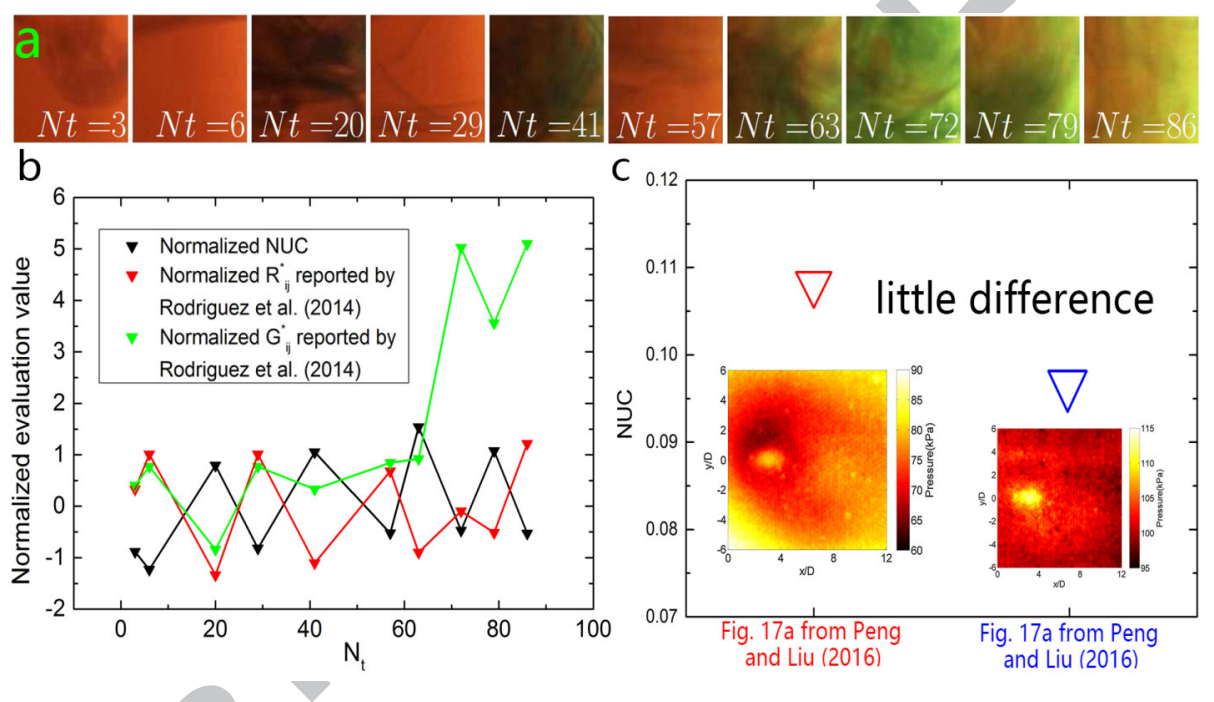

Figure 6: Other real-world applications: (a) concentration field reported by Rodriguez et al. (2014); (b) comparison result for above fields; (c) pressure fields from Peng and Liu (2016) and their evaluation values of distribution uniformity. 
Highlights

- A novel approach based on statistical measure which could efficiently and quantitatively measure the distribution uniformity of temperature was proposed.

- Effectiveness of the new method is demonstrated on both the presented temperature field data and the real open data in literature.

- It is shown that this technique can be expanded and adapted to analysis other temperature fields or physical fields. 